\title{
Seasonal Adjustment of China's Monthly Data to Take into Account the Effect of Mobile Holidays
}

\author{
Yanfei Song \\ Economics School, Jinan University, Guangzhou, China \\ Email: fiona9508@163.com
}

How to cite this paper: Song, Y.F. (2020) Seasonal Adjustment of China's Monthly Data to Take into Account the Effect of Mobile Holidays. Open Journal of Statistics, 10, 706-718.

https://doi.org/10.4236/ojs.2020.104044

Received: August 5, 2020

Accepted: August 23, 2020

Published: August 26, 2020

Copyright $\odot 2020$ by author(s) and Scientific Research Publishing Inc. This work is licensed under the Creative Commons Attribution International License (CC BY 4.0).

http://creativecommons.org/licenses/by/4.0/

(c) (i) Open Access

\begin{abstract}
Based on the X-13-ARIMA-SEATS model, aiming at the problem of mobile holidays in China's economic data, this paper introduces a new method of seasonal adjustment based on the AICC criterion to objectively select the parameters of dummy variables of mobile holidays. Taking the current total value of China's import and export as an example, we expound a new method for seasonal adjustment of mobile holidays such as Spring Festival, Dragon Boat Festival and Mid-Autumn Festival. Finally, the model is used to predict the total value of China's import and export in and out of the sample. The prediction results show that the relative error of the out of sample data is less than $5 \%$. The new method has advantages in the processing of macroeconomic data.
\end{abstract}

\section{Keywords}

X-13-ARIMA-SEATS, Seasonal Adjustment, Mobile Holiday Effect

\section{Introduction}

\section{Research Background}

In order to better monitor the operation of the national economy, it is necessary to scientifically process the economic data, especially the monthly and quarterly high-frequency data. Developed countries generally use the seasonally adjusted data as the basis for economic analysis and forecasting. Most monthly and quarterly macroeconomic data are influenced by seasonal factors. Taking the total value of import and export in the current period ( $\$ 10$ billion) as an example, the "advance of export and delay of import" could theoretically lead to a higher export growth rate and a lower import growth rate in the month during the Spring Festival. The purpose of seasonal adjustment of monthly data and quarterly data 
is to eliminate the data fluctuation caused by seasonal factors and make the data in each period at an "equal" level. The sequential growth rate calculated by this method can more timely reflect the potential trend of economic indicators. In addition, seasonal adjustment is also conducive to the observation of the current short-term indicators of the annual trade situation, and timely identifies the root cause of the problem, to do a good job in foreign trade response strategy.

Generally speaking, conventional seasonal factors include trend-period factors and seasonal components. However, the calendar effect of traditional Chinese festivals also has an important impact on the original data, especially the Spring Festival in China. The time of the lunar New Year on the Gregorian calendar occurs between January 21 and February 20 each year, which will cause some distortion in the data of monthly year-on-year growth rate. If the Spring Festival of the adjacent two years does not happen in January or February at the same time, the volatility of year-on-year data may be very large. At present, the latest outcome is the X-13-ARIMA-Seats, which is supported by the bank of Spain and developed by the census bureau. Based on the latest version of X-12-ARIMA (proposed by US Census Bureau's X-12-ARIMA for seasonal adjustment), this program adds the TRAMO/SEATS seasonal adjustment procedure, which has been widely used in the economic analysis process of central Banks and research institutions of various countries. Despite that the X-12-ARIMA regARIMA in program used in the pretreatment of the raw data of the adjustment module can be achieved, which eliminate the calendar effect (such as trading day effect, mobile holiday effect, etc.), but most of the built-in program X-12-ARIMA designed aiming at the condition of the western countries, such as Easter, Thanksgiving, for China the Spring Festival, the Dragon Boat Festival, Mid-Autumn Festival and so on did not provide a direct calculation program. In addition, in the existing seasonal adjustment studies considering the effect of mobile holidays in China, the pre-holiday, mid-holiday and post-holiday influence periods are selected in a subjective way, and the same influence periods are adopted for different time series. Therefore, there is a lack of relevant studies on the possible effects of different mobile holidays on different time series data. In view of this, this paper takes the current total value of China's import and export (RMB 10 billion) data as an example, and based on the X-13-ARIMA-SEATS program package, designs a direct calculation program for the Spring Festival, Dragon Boat Festival and Mid-Autumn Festival, and makes seasonal adjustment to the monthly data of China. At the same time, in view of the Spring Festival effect, this paper selected the parameters before, during and after the Spring Festival according to the AICC criterion, obtained the seasonally adjusted data excluding the Spring Festival effect by using the R, and used the adjusted data for prediction.

\section{Literature Review}

The idea of seasonal adjustment of time series can be traced back to 100 years ago. In 1919, Persons explicitly proposed to divide the time series into four parts: 
trend, cycle, season and irregular components. Seasonal adjustments to time series data remove natural or socio-historical factors from the original data (monthly or quarterly), making the data comparable and correctly reflecting underlying trends and turning points in the economic cycle in macroeconomic forecasts. In 1954, Shiskin of the US census bureau first developed a program called X-1 that USES computers to adjust the seasons. In 1955 an improved seasonal adjustment procedure was announced, known as X-2; By 1965 the X-11 method had been developed. The X-11 method was the international seasonal adjustment method at that time, and was also the core of X-11-ARIMA and X-12-ARIMA.

Currently, the X-12-ARIMA model is one of the most popular models for seasonal adjustment. The most important feature of this method is that regARIMA can enhance the ARIMA modeling ability and model selection ability of time series through the pre-adjustment module, and can estimate the user customized regression effects, such as mobile holiday effect, day effect, etc. Seasonal adjustment procedures such as X-12-ARIMA have been used in a number of recent studies involving Chinese data. 3 In their factor-augmented VAR study, Fernald, Spiegel and Swanson [1] use X-12-ARIMA to adjust for seasonality in 29 Chinese economic time series, after removing Chinese New Year effects by averaging January and February observations. The use of seasonally adjusted Chinese data in current applied work and the growing appetite for adjusted data among Chinese policymakers (revealed by the 2011 decision by the NBS to publish seasonally adjusted figures for key series) provide an additional motivation to investigate the application of seasonal adjustment procedures to Chinese time series. Although the X-12-ARIMA model has many advantages, some scholars still question it, that is, the X-12-ARIMA model is based on empirical methods and lacks strict statistical theoretical basis. Therefore, Gomez and Miraval [2] of the bank of Spain proposed the TRAMO/SEATS program based on the statistical model. Among them, the TRAMO program preadjusts the time series data, deals with the mobile holiday effect and outliners, and then uses the SEATS program to construct the ARIMA model and decompose the sequence. The X-12-ARIMA model adopts pre-defined filtering for the pre-adjusted data in the construction of the ARIMA model, while the SEATS program uses the ARIMA model for direct signal extraction to decompose the data into trend, seasonal and irregular factors. This decomposition assumes that all components are orthogonal and that the trend and seasonal components capture the permanent characteristics of the time series.

At present, one of the latest research results in the field of seasonal adjustment is to combine the latest version of the X-12-ARIMA model and the latest version of the TRAMO/SEATS model into a program package, namely the X-13-ARIMS-SEATs program package. X-13-ARIMA-SEATS based on TRAMO selects the model automatically. This method combines the advantages of the two methods based on experience and model. Since the program also provides a Genhol program to generate the mobile holiday regression factor, it effectively solves the problem of 
mobile holiday in seasonal adjustment. However, Genhol mainly aims at the mobile holidays in western countries, such as Easter and Thanksgiving, etc., but it does not provide a direct calculation program for the mobile holidays in China, such as Dragon Boat Festival and Mid-Autumn Festival. Although the date files of Chinese Spring Festival in previous years can be called up in the seasonal package of $\mathrm{R}$ program, only a fixed period can be selected for the influence period before, during and after the Spring Festival on the time series, which is the main reason why most studies have fixed the influence period of the Spring Festival. For the seasonal adjustment of the effect of Chinese New Year, representative domestic studies include: in the analysis of the seasonal adjustment of Chinese CPI data, He and Liu gave three solutions to the effect of Chinese New Year. In scheme 1 , the influence period before, during and after the festival was 7 days. In scheme 2 , the pre-festival, mid-festival and post-festival influence periods were 14 days, 7 days and 14 days respectively. In scheme 3, the pre-festival, mid-festival and post-festival influence periods are 20 days, 7 days and 20 days respectively. According to the comprehensive indicator Q value, in scheme 2 and scheme 3 were selected. LuanHuiDe and Zhang Xiao portal the analysis of Chinese social total retail sales of consumer goods, to set the effect of the Spring Festival for the affected period for 20 days before and during the festival the impact period for 7 days, post-holiday effect period for 20 days.

Although the paper also mentioned the need according to some indicators to choose the length of the subinterval, but it gave no definite index selection criteria. Similarly, Qun-Yong Wang and $\mathrm{Wu} \mathrm{Na}$, in the study of seasonally adjustment for China's social consumer goods retail sales monthly data, considered many mobile holiday (the Spring Festival, the Mid-Autumn Festival, Dragon Boat Festival, etc.) effect and holiday effect, etc., but in the analysis of the Spring Festival effect, the number of days before, during and after the festival are set to 20 days, 5 days, and 10 days, which is still subjective. Since different time series may be affected by the mobile holiday effect differently, it is necessary to use reasonable indicators to judge the influence period of the mobile holiday effect objectively. In view of this, Roberts and White (2015) [3] use AICC criterion to objectively select the influence period of the Spring Festival effect. In this paper, to be specific, X-13-ARIMA-SEATS program package is used to adjust China's current value of import and export data as an example to seasonally adjust the time series data of China and France.

\section{The Principle and Method of Seasonal Adjustment}

Based on the principle of seasonal adjustment, we will discuss how to introduce the regression variables of outliers, stock trading days and China mobile holiday effect into X-13ARIMA-SEATS program.

\subsection{Basic Principles of Seasonal Adjustment}

Essentially, seasonal adjustment is the estimation and elimination of seasonal 
variation factors from the original sequence. Its purpose is to better reveal the potential characteristics of the monthly (or quarterly) sequence, so as to truly reflect the time series' variation rules. The monthly (or quarterly) data sequence is usually broken down into seasonal components, trend cyclical components and irregular components. Multiplication model is most widely applied in the macroeconomic indicators, decomposition equation can be expressed as $Y_{t}=S_{t} \times T C_{t} \times I_{t}$; $Y_{t}, S_{t}, T C_{t}$ and $I_{t}$ are seasonal ingredients in original sequence, trend cycle component, and no rules into points respectively. The seasonally adjusted sequence $S A_{t}=T C_{t} \times I_{t}$ was obtained by removing the seasonal component from the original sequence, and the sequential growth rate was calculated accordingly, with the formula as follows:

$$
g_{t}=\frac{S A_{t}-S A_{t-1}}{S A_{t-1}} \times 100 \% .
$$

In this paper, the seasonal adjustment of the money supply is based on the us census bureau procedure X-13ARIMA-SEATS. The basic principle of the X13ARIMA-SEATS program can be in three stages: RegARIMA model is first established to carry out of sample prediction of the sequence for one year in front and back direction to supplement the data; Then, a series of filters of different lengths were repeatedly used for moving average operation, and various components (seasonal components, trend recycling components and irregular components, etc.) were extracted. Finally, the results of seasonal adjustment were examined.

RegARIMA model can be expressed as:

$$
\varphi(L) \phi\left(L^{s}\right)(1-d)^{d}\left(1-L^{S}\right)^{D}\left(y_{t}-\sum \beta_{i} x_{i t}\right)=\theta(L) \Theta\left(L^{s}\right) \varepsilon_{t} .
$$

Among them is the lag operator $L, s$ is the seasonal cycle, $\varphi(L)$ and $\phi\left(L^{s}\right)$ is a season and season since back to operator respectively, $\theta(L)$ and $\Theta\left(L^{s}\right)$ is a season and moving average operator respectively, $d$ and $D$ respectively the number of seasonal difference and the seasonal difference. $s \varepsilon$ is white noise process. $y_{t}$ as the original sequence or on the original sequence after the exponential sequence, $x$ including constant term, outliers, trading effect and mobile holiday effect, etc. the introduction methods of outliers, trading day effect and mobile holiday effect in the model are introduced in the following sections.

\subsection{Identification Method of Outlier Effect}

The X-13-Arima-Seats program can automatically detect four types of outliers, namely, point outliers (AO), horizontal drift (LS), temporary change (TC), and seasonal outliers (SO). AO represents the outlier point at a certain time; LS means that a variable changes instantaneously from a certain point to a new level and stays there; TC means that the variable changes to a new level instantly at a certain time and then returns to the original water level through exponential decay. It means that seasonal patterns change after a certain point. The program calculates the $t$ statistics corresponding to each outlier type for all the time points 
in the detection interval of outliers. Determine whether to add the outlier variable to the model based on its significance. It should be noted that the program must recognize the outliers automatically, and only those that can be given a reasonable explanation (e.g. social unrest, changes in government policy, economic crisis, etc.) can be incorporated into the RegARIMA model as a regression factor. When outliers are too concentrated in some fixed month, it usually means that there is a mobile holiday effect in that month and must be overcome. In addition, the fewer outliers there are, the more concise the model tends to be.

\subsection{Day Effect Treatment}

Economic variables change regularly over seven days a week. For example, businesses usually conduct accounting transactions on working days (Monday to Friday), and residents are accustomed to withdrawing cash from Banks at weekends for purchases. Thus, as an indicator of stock, the money supply is measured in relation to the corresponding week. Considering this special effect, the following six dummy variables are set as regression variables to overcome the trading day effect:

$$
D_{1, t}=\left\{\begin{array}{lc}
1 & \text { The } \varpi \text { th day of month } t \text { is Monday } \\
-1 & \text { The } \varpi \text { th day of month } t \text { is Sunday } \\
0 & \text { others }
\end{array}\right.
$$

where $\varpi$ is the smaller values between the observation registration date (correspond to Gregorian calendar date) and the length of the month.

\subsection{Handling of Mobile Holidays}

Mobile holidays are holidays that occur regularly but do not necessarily correspond to a fixed Gregorian calendar time each year. For example, Chinese Spring Festival, Mid-Autumn Festival and Dragon Boat Festival correspond to fixed lunar dates, but their corresponding Gregorian calendar dates are variable. The stock data are closely related to the observation registration date, which corresponds to the Gregorian calendar date. Because of the mobility of holidays, this difference cannot be eliminated by the moving average method of the seasonally adjusted process.

The Spring Festival is the most solemn traditional festival of the Chinese nation. During the Spring Festival, Chinese people leave their jobs to reunite with their families, which is accompanied by mass migration. For the export sector, the Spring Festival effect is also obvious. Due to the large number of employees returning home, many export enterprises will "jump the gun" before the festival to expand exports, until the seven-day Spring Festival holiday or even after the 15-day Lantern Festival to return to normal. Therefore, how to eliminate the "Spring Festival effect" in the monthly data has become a special research field.

To eliminate the Spring Festival effect, dummy variables should be constructed as follows: 


$$
H_{i}\left(\tau_{i}, t\right)=\frac{\tau_{i t}}{\tau_{t}}, i=1,2,3 .
$$

$i=1,2,3$ respectively first, section, mart, $\tau_{i t}$ is the number of days affected by part $i$ in month $t$ in February. For better understanding above formula, let's give an example, if the $\tau_{1}=\tau_{2}=\tau_{3}=5$, namely, the number of days that affect the time series data before, during and after the Spring Festival are all 5 days, and assumes that year to January 31, the day before the Spring Festival, the first effect into the number of days to five days in January, therefore,

$$
\begin{aligned}
& H_{1}\left(\tau_{1}, 1\right)=1, H_{1}\left(\tau_{1}, 2\right)=0, \cdots, H_{12}\left(\tau_{1}, 12\right)=0 \\
& H_{2}\left(\tau_{2}, 1\right)=0.2, H_{2}\left(\tau_{2}, 2\right)=0.8, \cdots, H_{2}\left(\tau_{1}, 12\right)=0 \\
& H_{3}\left(\tau_{2}, 1\right)=0, H_{3}\left(\tau_{3}, 2\right)=1, \cdots, H_{3}\left(\tau_{3}, 12\right)=0
\end{aligned}
$$

Three dummy variables were constructed to eliminate the influencing factors of the Spring Festival. Ever for the Spring Festival holiday, the study usually affects the number of days to constant processing. Of all time series variable without area, choose the same subinterval is obviously undesirable influence length, without considering the different characteristics of different. With reference to the method of Roberts and White (2015) [3], the number of days affected by the festival before and during the festival is 0 - 20 days, and the relevant programs are called cyclically and selected according to the AICC minimum criterion. Specifically, the AICC specifications applicable to limited sample sizes can be listed as follows:

$$
\mathrm{AICC}=-2 \mathcal{L}_{N}+2 p \frac{1}{1-(p+1) /(T-12 D-d)} .
$$

Among them, $\mathcal{L}_{N}$ is the value of the log-likelihood function of the estimated parameter, $p$ is the number of estimated parameters, and $D$ is the seasonal difference, and $d$ is the order of conventional difference.

The Dragon Boat Festival and the Mid-Autumn Festival are also traditional Chinese festivals. According to statistics, these two traditional festivals do not fall in the same month every year, and they cannot be eliminated through regular seasonal adjustment. Therefore, it is necessary to regard ima modeling stage to eliminate the original sequence by constructing regression variables before the regular seasonal adjustment of the current import and export volume. In order to determine whether there is the Dragon Boat Festival and mid autumn festival-to the time sequence effect, we firstly determined the three parameters of the Spring Festival effect $t$ based on the package of $\mathrm{R}$ language, and then we designed a direct the application of the Dragon Boat Festival and Mid-Autumn Festival, to determine if no significant effect on a virtual variable, then move to should be a holiday.

\section{Empirical Results}

This paper selected 2008 m1-2017 m12 (the Chinese Mid-Autumn Festival and Dragon Boat Festival started in 2008) as the seasonally adjusted sample range of 
the total import and export amount in the current period (tens of billions of dollars), and the data of 2018 m1-2018 m12 was reserved for comparison with the predicted value of the model.

\subsection{Seasonal Adjustment of Current Export Value}

According to the AICC criterion, the model finally chooses the pre-festival influence period of 6 days, the mid-festival influence period of 6 days, and the post-holiday impact period is 12 days, which maximize the value of AICC. The ARIMA model selected by the ndiffs function in $\mathrm{R}$ is $(0,1,0)(0,1,1)$. Table 1 shows the result of regARIMA fitting of current value after logarithmic conversion by nidify program.

The results above show that the effects of pre-festival (xreg1), mid-festival (xreg2) and post-festival (xreg3) are significant. At the same time, it can be seen from the symbols of the parameters that pre-festival has a positive impact on the export, while the overall export value is negatively affected by the mid-festival and post-festival. It is consistent with the phenomenon that enterprises "jump the gun" to expand the export before the pre-festival. In addition, the trading day effect is significant, but the Dragon Boat Festival effect (xreg4) and the Mid-Autumn Festival effect (xreg5) are not significant. In addition, the model identified February 2012 as AO outliers. Therefore, the last seasonal adjustment model excludes the Mid-Autumn Festival effect and the Dragon Boat Festival effect.

Table 2 is the main statistics of the model adjusted for the last quarter of the total value of the current period after logarithmic conversion, and Table 3 is the QS statistics of the seasonally adjusted diagnostic test. QS is a kind of statistic. The null hypothesis is that there is no seasonal effect in the series, which is suitable for testing whether there is still seasonal effect in the time series after modeling

Table 1. regARIMA fitting results of current total value of exports.

\begin{tabular}{ccccc}
\hline variable & Estimate & Std. Error & Z Value & $\operatorname{Pr}(>|\mathrm{Z}|)$ \\
\hline Weekday & 0.00372 & 0.00915 & 4.062 & $4.87 \mathrm{e}-05^{* * *}$ \\
Xreg1 & 0.147 & 0.0199 & 7.364 & $1.78 \mathrm{e}-13^{* * *}$ \\
Xreg2 & -0.104 & 0.0228 & -4.563 & $5.04 \mathrm{e}-06^{* * *}$ \\
Xreg3 & -0.2673 & 0.0269 & -9.953 & $<2 \mathrm{e}-16^{\star * *}$ \\
Xreg4 & -0.0226 & 0.0144 & -1.570 & 0.117 \\
Xreg5 & 0.0028 & 0.0156 & 0.182 & 0.856 \\
AO2012. Feb & -0.154 & 0.0376 & -4.082 & $4.47 \mathrm{e}-05^{* * *}$ \\
MA-Seasonal-12 & 0.754 & 0.0671 & 11.232 & $2 \mathrm{e}-16^{*}$ \\
\hline
\end{tabular}

Table 2. Main statistics of the last seasonally adjusted model of exports.

\begin{tabular}{ccccc}
\hline AICC & BIC & QS & The Box-Ljung & Shapiro \\
\hline 270 & 296.6 & 0 & 0 & 0.992 \\
\hline
\end{tabular}


and seasonal adjustment. The results show that there is a seasonal and significant seasonal effect in the original chronological sequence of the total output value of the current period, the residual of RegARIMA model has no seasonal effect, and the final sequence after seasonal adjustment has no seasonal effect. Finally, by plotting the seasonally adjusted data with the original total amount of import and export in the current period in Figure 1, we can find that the seasonal factors are well removed after seasonal adjustment.

\subsection{Forecast of Total Export Value}

According to the selected final model, the total export value in the current period is predicted, and the forecast time range is from January 2018 to December 2018. Figure 2 shows the comparison between the predicted value and the real value, and it can be seen that the predicted value and the real value trend are consistent. Table 4 is the error table of the prediction phase pairs. The results in Table 4 show that the relative error in other months is small except that the relative error in December reaches 5\%, and the total average relative error is $2.22 \%$.

Table 3. QS statistics of seasonally adjusted diagnostic tests of exports.

\begin{tabular}{ccc}
\hline copy & statistic & P-value \\
\hline The Original Series & 68.70 & 0 \\
The Original Series adj (EV) & 162.84 & 1 \\
Residuals & 0.00 & 1 \\
Seasonally Adjusted Series & 0.00 & 1 \\
The Seasonally Adjusted Series (EV adj) & 0.00 & 1 \\
Irregular Series & 0.00 & 1 \\
\hline Irregular Series adj (EV) & 0.00 & 1 \\
\hline
\end{tabular}

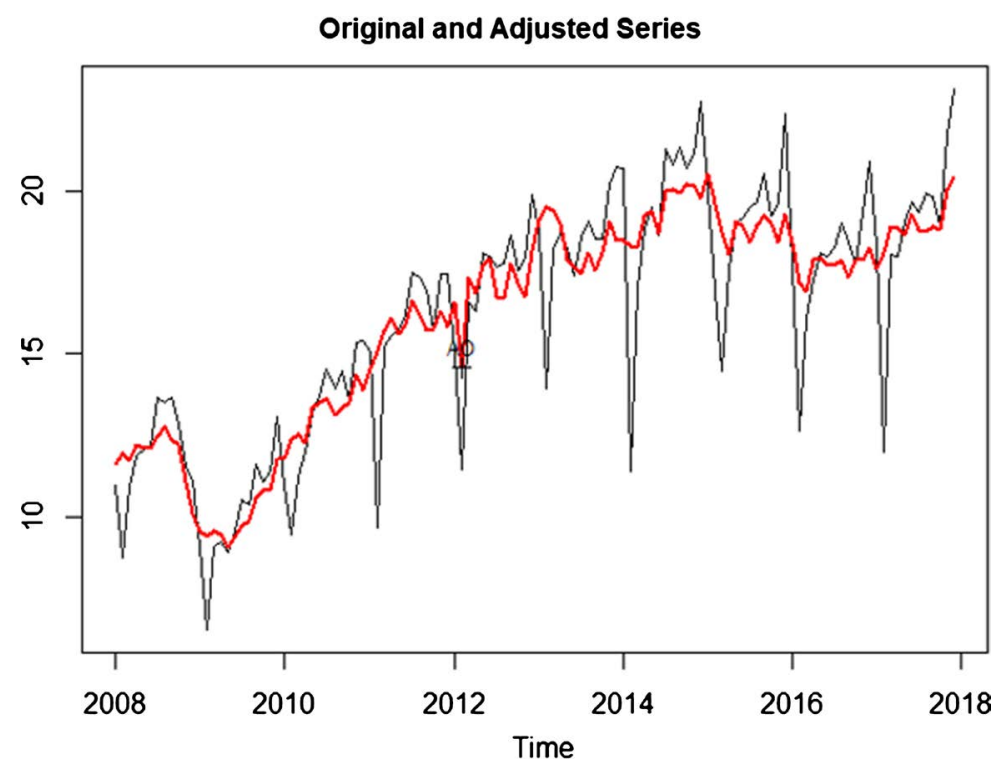

Figure 1. Total export value and seasonally adjusted value (tens of billions of dollars). 


\subsection{Seasonal Adjustment of Current Total Import Value}

According to the AICC criterion, the model finally chooses the pre-festival influence period of 3 days and the mid-festival influence period of 9 days, and the post-festival effect is not significant. The ARIMA model selected by the ndiffs function in $\mathrm{R}$ is $(0,1,0)(0,1,1)$ Table 1 shows the result of regARIMA fitting of current value after logarithmic conversion by ndiffs program (Table 5 ).

The results show that the effect of xreg1 and xreg2 before the Spring Festival is significant. At the same time, it can be seen from the parameter symbols that the total value of import in the current period is the same as that of export, and the positive effect is brought to export before the festival, while the negative effect is brought to the total value of import during the festival and xreg2. In addition,

Table 4. Relative errors of prediction of exports.

\begin{tabular}{cccc}
\hline Time & Predictive value & The relative error & The relative error \\
\hline Jan 18 & 20.30 & 20.05 & 0.013 \\
Feb 18 & 16.47 & 17.16 & 0.040 \\
Mar 18 & 17.23 & 17.41 & 0.010 \\
Apr 18 & 20.14 & 20.00 & 0.0060 \\
May 18 & 21.38 & 21.29 & 0.0042 \\
Jun 18 & 21.06 & 21.66 & 0.028 \\
Jul 18 & 21.96 & 21.26 & 0.019 \\
Aug 18 & 22.47 & 21.74 & 0.034 \\
Sep 18 & 21.93 & 22.67 & 0.033 \\
Oct 18 & 21.27 & 21.72 & 0.026 \\
Nov 18 & 23.20 & 22.74 & 0.020 \\
Dec 18 & 23.40 & 22.12 & 0.057 \\
Mean relative error & & 0.022 & \\
\hline
\end{tabular}

Table 5. regARIMA fitting results of current value of imports after logarithm conversion.

\begin{tabular}{cccc}
\hline Variable & Std. Error & $\mathrm{Z}$ value & $\operatorname{Pr}(>|\mathrm{Z}|)$ \\
\hline Weekday & 0.12 & 9.34 & $<2-16^{* * *} \mathrm{e}$ \\
Xreg1 & 0.62 & 1.98 & $0.0473^{\star}$ \\
Xreg2 & -2.88 & -6.28 & $3.37 \mathrm{e}-10^{* * *}$ \\
Xreg4 & -0.22 & -1.00 & 0.31718 \\
Xreg5 & -0.49 & -2.038 & $0.04155^{\star}$ \\
LS2008. Dec & -2.68 & -3.98 & $8.25 \mathrm{e}-05^{* * *}$ \\
AO2011. Jan & 2.08 & 4.09 & $4.29 \mathrm{e}-05^{* * *}$ \\
AO2013. Mar & 2.48 & 5.18 & $2.21 \mathrm{e}-07^{* * *}$ \\
MA-Nonseasonal & 0.23 & 0.093 & $0.00335^{*}$ \\
MA-Seasonal & 0.47 & 0.081 & $6.12 \mathrm{e}-09^{*}$
\end{tabular}


day effect and Mid-Autumn Festival effect (xreg5) significant. In addition, the model identified January 2011 and March 2013 as AO outliers. Therefore, according to the above results, the last seasonal adjustment model was added before the Spring Festival, after the Spring Festival and the effect of the Dragon Boat Festival. Table 6 shows the main statistics of the last seasonally adjusted model of the total output value of the current period after logarithmic conversion, and Table 7 shows the QS statistics of the seasonally adjusted diagnostic test.

The results show that the original time series of export current value has seasonal and significant seasonal effect, the residual of regAERIMA model has no seasonal effect, and the final sequence after seasonal adjustment has no seasonal effect. Finally, the seasonally adjusted data and the original total data of the inlet and outlet in the current period are plotted in Figure 2 and Table 8.

Table 6. Main statistics of the last seasonally adjusted model of imports.

\begin{tabular}{ccccc}
\hline AICC & BIC & QS & The Box-Ljung & Shapiro \\
\hline 252.9 & 296.6 & 0 & 0 & 0.988 \\
\hline
\end{tabular}

Table 7. QS statistics of seasonally adjusted diagnostic tests of imports.

\begin{tabular}{ccc}
\hline copy & statistic & P-value \\
\hline The Original Series & 68.89 & 0 \\
The Original Series adj (EV) & 161.14 & 0 \\
Residuals & 0.000 & 1 \\
Seasonally Adjusted Series & 0.000 & 1 \\
The Seasonally Adjusted Series (EV adj) & 0.000 & 1 \\
Irregular Series & 0.000 & 1 \\
Irregular Series adj (EV) & 0.000 & 1 \\
\hline
\end{tabular}

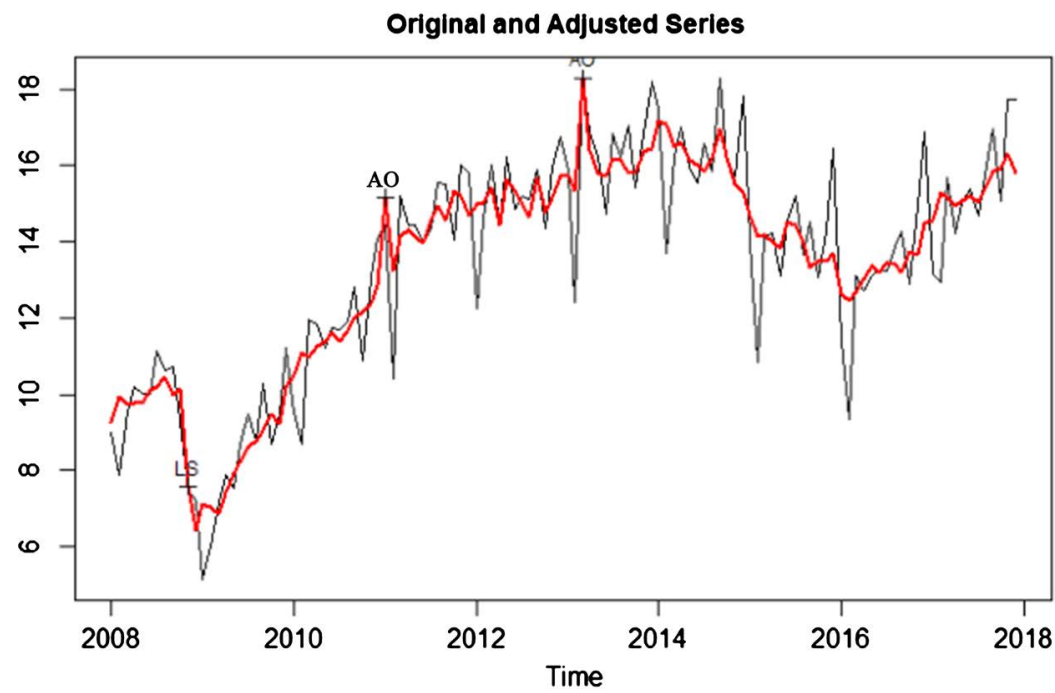

Figure 2. Total export value and seasonally adjusted value (tens of billions of dollars). 
Table 8. Relative errors of prediction of imports.

\begin{tabular}{cccc}
\hline time & Predictive value a & The relative error & The relative error \\
\hline Jan 18 & 17.048 & 18.01 & 0.044 \\
Feb 18 & 13.082 & 13.79 & 0.041 \\
Mar 18 & 17.23 & 17.91 & 0.038 \\
Apr 18 & 15.88 & 15.17 & 0.047 \\
May 18 & 18.15 & 18.79 & 0.034 \\
Jun 18 & 17.87 & 17.51 & 0.020 \\
Jul 18 & 18.49 & 18.75 & 0.015 \\
Aug 18 & 19.26 & 19.95 & 0.015 \\
Sep 18 & 19.86 & 19.50 & 0.019 \\
Oct 18 & 19.99 & 18.33 & 0.091 \\
Nov 18 & 18.15 & 18.27 & 0.0064 \\
Dec 18 & 16.69 & 16.42 & 0.15 \\
\hline
\end{tabular}

\section{Conclusions}

In order to better monitor the operation of the national economy, it is necessary to scientifically process the economic data, especially the monthly and quarterly high-frequency data. Developed countries generally use the seasonally adjusted data as the basis for economic analysis and forecasting. China has developed a version of X-12-ARIMA software and NBS-SA software for seasonal adjustment of economic data, but because the software is not made public, it is not known whether the different economic indicators use reasonable standards to distinguish. The existing studies on seasonal adjustment of China's economic data all adopt a fixed influence period when dealing with the effect of mobile holidays, and do not differentiate different economic indicators, which ignores the fact that there may be different characteristics among indicators, and there will inevitably be deviations in the prediction.

According to Roberts and White (2015) [3] the latest research results, we used AICC criterion on objective selection of the Spring Festival effect, based on China's gross value of import and export, in the use of X-13-ARIMA-SEATS. We finally designed a direct application to make seasonally adjustment for China's monthly data of the Spring Festival, the Dragon Boat Festival, Mid-Autumn Festival. In addition, in view of the Spring Festival effect, this paper selected the parameters before, during and after the Spring Festival according to the AICC criterion, obtained the seasonally adjusted data excluding the Spring Festival effect by using the $\mathrm{R}$ sequence, and used the adjusted data for prediction. The results show that the total value of exports in the three periods of the Spring Festival has a significant holiday effect, while the total value of imports in the current period has only a significant holiday effect before and during the Spring Festival. In ad- 
dition, the Dragon Boat Festival effect and the Mid-Autumn Festival effect of the current total value of exports are not significant, while the import of the current total value of the Mid-Autumn Festival effect is significant. Finally, this paper forecasts the total value of imports and exports in the current period from the out-of-sample data, and the relative error of the prediction is less than $5 \%$, indicating that the accuracy of the model is relatively high. Therefore, the method of using AICC criterion to select the parameters of mobile holiday dummy variable has great advantages.

\section{Conflicts of Interest}

The author declares no conflicts of interest regarding the publication of this paper.

\section{References}

[1] Fernald, J., Spiegel, M.M. and Swanson, E.T. (2014) Monetary Policy Effectiveness in China: Evidence from a FAVAR Model. Federal Reserve Bank of San Francisco Working Paper No 2014-07. https://doi.org/10.3386/w20518

[2] European Central Bank. Seasonal Adjustment of Monetary Aggregates and HICP for the Euro Area. http://www.ecb.int/pub/pdf/other/sama0008en.pdf

[3] Roberts, I. and White, G. (2015) Seasonal Adjustment of Chinese Economic Statistics. Research Discussion Paper, Reserve Bank of Australia, Sydney. 